\title{
6. ZUSAMMENFASSUNG UND ERGEBNISSE
}

Geschichten über Scharen von Dämonen, über wunderbare Errettungen und die ohrfeigende Gottesmutter Maria in Guiberts Werken bilden den Ausgangspunkt der vorliegenden Studie. Die Forschung tat sich schwer mit diesen Berichten über Eingriffe überirdischer Mächte in Guiberts Welt, denn sie scheinen schlecht in das Bild des kritischen, rationalen Menschen zu passen, das sie von diesem Abt aus dem 12. Jahrhundert entworfen hat. Die Wundererzählungen in den Schriften Guiberts de Nogent sind deshalb bis heute nur am Rande betrachtet worden. Die vorliegende Studie hat sich zum Ziel gesetzt, den Umgang Guiberts de Nogent mit Wundern und Wundererzählungen darzustellen. Sie will zudem aufzeigen, welche Funktionen und Bedeutungen ihnen in der intellektuellen Gemeinschaft zukamen, in der sich Guibert als Mönch in Saint-Germer-de-Fly und als Abt von Nogent bewegte.

\section{Deutungen}

Als erster Aspekt des Umgangs mit dem Wunder steht im zweiten Kapitel Guiberts Deutung von Ereignissen als Wunder im Zentrum. Darin wurde herausgearbeitet, daß diese Deutungen zum einen von autoritativen Texten geprägt sind, zum anderen, daß Guiberts Deutungsarbeit in Zusammenhang steht mit Diskussionen über das Phänomen Wunder, die unter kirchlichen Würdenträgern geführt wurden.

Guibert unternimmt im Reliquientraktat eine konzeptionelle Annäherung an das Phänomen Wunder. Obwohl er seine Überlegungen im Laufe einer Abhandlung über den Reliquienkult anstellt, wird deutlich, daß Wunder für Guibert nicht nur an die Vermittlung von Heiligen gebunden sind, sondern $\mathrm{da}$ er sie auf breiterer Ebene als Zeichen der Gnade Gottes betrachtet. Die Begrifflichkeit für diese Zeichen ist dementsprechend flexibel. Als Deutungsschemata zur Einordnung dieser Zeichen sind für Guibert die biblischen Wunder wichtig, wie dies im Mittelalter durchaus üblich war. Guibert verwendet neben den alttestamentarischen Wundern vor allem die zentralen Mysterien des christlichen Glaubens: die jungfräuliche Geburt Christi, seine Auferstehung und Präsenz im Sakrament der Eucharistie. Die neutestamentarischen Wunder Christi nehmen einen weniger wichtigen Platz ein.

Guibert strebt nicht eine Erklärung des Phänomens Wunder an. Als Konzept genügt ihm der augustinische Wunderbegriff. Guibert wählt einen anderen Zugang zum Wunder und versucht Rahmenbedingungen für diese Zeichen der Gnade Gottes festzulegen. In erster Linie untersucht er die moralische Disposition von Mittlern und Empfängern von Wundern. Dies kann im $\mathrm{Zu}$ sammenhang damit gesehen werden, daß Guibert sich auch als Exeget auf die Auslegung der biblischen Schriften nach dem moralischen Schriftsinn konzentriert. Anhand von Erörterungen einzelner Fälle stellt er allgemeinere Überlegungen an, unterscheidet jedoch nicht genau zwischen Empfängern und Mitt- 
lern von Wundern. Als Rahmenbedingungen für Empfänger sind vielfältige Konstellationen genannt, unter denen ein Wunder geschehen kann. So werden Wunder durch unschuldige Kinder, aufgrund von Buße und Reue, aber auch für Ungläubige gewirkt. Erst bei der Erörterung der Heiligenkulte, in deren Zusammenhang Wunder beurteilt werden müssen, entwickelt Guibert griffigere Zugangsweisen und weist auf Mißbräuche hin. Unter anderem betont er die Wichtigkeit der Anerkennung von Wundern durch die kirchliche Autorität. Damit ist Guibert nicht allein. Solche Überlegungen wurden auch im anglonormannischen Bereich im Zuge der kirchlichen Neuordnung Englands nach der normannischen Eroberung angestellt. Davon zeugen Schriften des Lanfranc, des Anselm und auch des Eadmer von Canterbury.

Im zweiten Teil des Kapitels wird aufgezeigt, daß Guiberts Erörterung über Wunder in Zusammenhang steht mit Diskussionen über Heilige und Wunder, die unter kirchlichen Würdenträgern geführt wurden. Diese Würdenträger waren, wie kirchenrechtliche Quellen immer wieder betonen, mit der Kontrolle über den christlichen Kult beauftragt. Wunder spielten eine wichtige Rolle für die Anerkennung von Heiligen. Diese Anerkennung wurde zu Anfang des 12. Jahrhunderts in erster Linie auf der Ebene der Diözese oder der Kirchenprovinz vorgenommen. Bischofsurkunden aus den Diözesen der Kirchenprovinz Reims zeugen davon, daß sich der Klerus jährlich ein bis zweimal am Bischofshof versammelte. Heilige wurden auch vor der Durchsetzung des päpstlichen Kanonisationsverfahrens nicht nur >von unten<, per viam cultus, kanonisiert, sondern kirchliche Autoritätspersonen versuchten, über die Anerkennung von Heiligen an den Synoden den Kult zu kontrollieren. Am Beispiel des Arnulf von Soissons wurde gezeigt, daß den Bischöfen dabei nicht nur eine passive Rolle zukam, sondern da 3 die Anerkennung der Kirchenversammlung nötig sein konnte, um Heiligenkulte in die Wege zu leiten.

Wunder wurden aber auch im Rahmen der Kontrolle der Kultausübung durch kirchliche Würdenträger diskutiert. So weisen Guiberts Werke, wie auch hagiographische Schriften darauf hin, daß im Rahmen dieser Funktion unter Bischöfen und Äbten über Wunder als außergewöhnliche Vorfälle beraten wurde und daß Zeugen gesucht wurden, die über solche Vorfälle aussagen konnten. Ein regulierender Zugriff in den Bereich des Reliquienkults und Wundergeschehens durch die kirchliche Autorität ist auch in diesem Bereich sichtbar.

\section{Verweise}

Im dritten Kapitel stehen die Verweise auf die Herkunft von Wundererzählungen in Guiberts Texten im Zentrum. Diese Verweise werden vom Verfasser angeführt, um die Glaubwürdigkeit des Berichteten zu garantieren. Verweise auf Zeugen der Erzählungen können zudem Diskussionszusammenhänge aufzeigen, in denen Wundererzählungen eine Rolle spielten. Der Vergleich von Konventionen der Glaubwürdigkeitsgarantien in historiographischen, hagiographischen und legendarischen Texten hat gezeigt, daß die Verweise auf die 
außertextliche Realität vor allem in historiographischen und in hagiographischen Texten, im speziellen in Wundersammlungen, ähnlich sind. Garantien der Glaubwürdigkeit müssen sowohl für politische oder institutionelle Ereignisse als auch für Wunder, als Zeichen der Gnade Gottes, geliefert werden. Diese Glaubwürdigkeit kann über zwei hauptsächliche Elemente hergestellt werden: über die Nennung von Zeugen und über Angaben zu Ort, Zeit und Akteuren des Geschehens.

Die Auswertung der Zeugenangaben in Bezug auf Kommunikationsnetze für Wundererzählungen hat gezeigt, da $B$ von Guiberts weltlichem, monastischem und weltklerikalem Netz das monastische für die Kommunikation von Wundererzählungen am bedeutendsten war. Wundererzählungen wurden innerhalb von monastischen Gemeinschaften thematisiert. Guibert gehörte als Mönch über lange Zeit der benediktinischen Gemeinschaft von Saint-Germer-de-Fly an. Die Erzählungen, von denen er aus Saint-Germer berichtet, zeigen, daß außergewöhnliche Vorfälle innerhalb der Gemeinschaft beurteilt und eingeordnet werden mußten. Diese Vorfälle wurden zudem in Form von didaktischen Erzählungen kommuniziert.

Zeugenangaben weisen, über Saint-Germer-de-Fly hinaus, auf ein weiter reichendes Netzwerk von benediktinischen Mönchen hin. So konnte beispielsweise im Falle der Santiagopilgerlegende, einer vielfach überlieferten Wiedergängerlegende, nachgewiesen werden, daß die Kommunikation dieser Geschichte in eine Gruppe um Anselm von Bec/Canterbury zu situieren ist, die mit Cluny in engem Kontakt stand. Die Zirkulation solcher Erzählungen in einem benediktinischen Netzwerk wird verständlich durch die hohe Mobilität von Mönchen und Handschriften in diesem Umkreis.

Als weitere wichtige Zeugen erwiesen sich Personen aus dem Weltklerus. Die Kleriker von Laon standen Guibert geographisch und institutionell nahe. Der Klerus der Diözese versammelte sich mindestens einmal pro Jahr am Bischofshof. Guibert berichtet von weiteren Beziehungen, die er zum Bischof und seinem Umfeld pflegte. Die genannten Zeugen aus dem Kreis der Bischöfe waren oft Vertreter einer reformorientierten Gruppe des regionalen Klerus. Denkbar ist, daß sich Guibert über Zeugennennungen vor allem zu diesem Kreis in Beziehung setzen wollte. Beziehungen Guiberts zu reformfreundlichen Bischöfen und Äbten gehen aber auch aus den Widmungen seiner Schriften hervor.

Guibert weist für einen Teil der Erzählungen explizit aus, ob er sich auf mündliche oder schriftliche Quellen beruft. So im Falle eines Marienmirakels, für das weitere Handschriften aus dem 11. und 12. Jahrhundert, Predigt- und Mirakelsammlungen aus der Region, beigebracht werden konnten. Die handschriftliche Überlieferung bestätigt Guiberts Angabe über das Medium der Bezeugung auch außerhalb seiner eigenen Texte. In anderen Fällen wird nicht präzisiert, ob Wundererzählungen mündlich oder schriftlich weitergegeben wurden. Intertextuelle Beziehungen $z u$ weiteren Schriften, die das gleiche Wunder überliefern, sind in diesen Fällen schwierig nachzuweisen. 
Die Untersuchung von Zeit-, Ort- und Akteurangaben hat gezeigt, daß der Dimension des Ortes in Guiberts Schriften am meisten Beachtung geschenkt wird. Oft sind Ereignisse im geographischen Raum verankert. Die Dimension der Zeit wird mit weniger Präzision gehandhabt. Das Gleiche gilt für die Akteure. Am ehesten sind die involvierten Kleriker als kirchliche Autorität genauer beschrieben. Die Verankerung im Raum zeigt, daß die Erzählungen zum größten Teil aus einem regionalen Umfeld stammen. Die Analyse der Orte wunderbaren Geschehens hat ergeben, daß Gräber als Orte der Heiligenverehrung keine überaus wichtige Rolle spielen. Hingegen werden Reliquien auf Reisen geschickt und erlangen so eine große Mobilität. Gräber sind aber in allgemeinem Sinne wichtig. Der Übergang vom Leben ins Jenseits wird in den Erzählungen oft thematisiert. Kirchen als Orte des Wundergeschehens und Versammlungsort der Gläubigen spielen ebenfalls eine wichtige Rolle. Das Wundergeschehen in Guiberts Schriften ist weniger an feste Örtlichkeiten gebunden. Dies mag damit zusammenhängen, daß Guibert sehr viele Marienwunder, aber auch Eucharistiewunder und Dämonenerzählungen in seine Schriften einfügt, die nicht an ein Grab als Kultort gebunden sind. Wichtig ist hingegen der öffentliche Raum als Ort des Wundergeschehens, welcher die Ereignisse überprüfbar macht.

Vergleicht man Zeugennennungen und referentielle Bezüge von Guiberts Wundererzählungen, zeigt sich, daß diese in einem regionalen Umfeld kommuniziert wurden. Dies gilt sowohl für die mündliche Überlieferung als auch für die erwähnten schriftlichen Zeugnisse. Wunderwirkende Heilige stammen aus einem regionalen Kontext, oft tritt aber auch die ortsunabhängige Maria als Wunderwirkerin auf.

In den ersten beiden Teilen der Untersuchung wurden zwei Formen der Annäherung Guiberts an das Phänomen des Wunders analysiert. Der erste Zugang hat die Anerkennung von Ereignissen als Wunder zum Inhalt. Dabei spielen Mittler und Begünstigte eine große Rolle. Die zweite zielt auf die Glaubwürdigkeit von Wundererzählungen über die Nennung von Zeugen. Diese beiden Zugänge können die Einschätzung eines wunderbaren Ereignisses erschweren, wenn sie zueinander in Widerspruch treten. In solchen Fällen löst Guibert in seinen Texten den Widerspruch auf, indem er der einen oder der anderen den Vorzug gibt oder sich mittels Ironie vom Berichteten distanziert.

\section{Funktionen}

Die Funktionen von Wundererzählungen in den verschiedenen Schriften Guiberts stehen im Mittelpunkt des vierten Kapitels. Eine wichtige Rolle spielen die Wundererzählungen in Guiberts autobiographischer Schrift, den Monodiae. Wie neuere Untersuchungen (Trudy Lemmers, Reinhold Kaiser) gezeigt haben, sind sie Teil eines Werkes, das bewußt komponiert wurde. Eine Analyse der Gesamtstruktur hat ergeben, daß die Wundererzählungen immer an Brüchen der Handlung eingefügt sind. In den autobiographischen Teilen stehen sie an Brüchen seines eigenen Lebens, im dritten Buch, der »Tragödie 
der Leute von Laon«, an Brüchen des dargestellten städtischen und diözesanen Geschehens. Die Wundererzählungen stehen in engem Zusammenhang mit dem Thema der Haupthandlung: sie variieren das Verhalten von anderen Personen in ähnlichen Situationen. Wenn Guiberts Stimme gemäß dem Titel des Werks, Monodiae, eine Stimme außerhalb des Chores darstellt, können die Wundererzählungen in den Exkursen als Chor gelesen werden.

Wundererzählungen in den Monodiae haben eine didaktische Funktion, sowohl für ein monastisches wie auch für ein weltliches Publikum. Wundererzählungen, die im monastischem Umfeld situiert sind, thematisieren das Verhalten Einzelner in der klösterlichen Gemeinschaft. Sie verurteilen die Geldgier und das Abweichen vom rechten Glauben, mahnen zu Buße und Beichte. Wundererzählungen aus dem weltlichen Umfeld thematisieren die gleichen Sünden, verurteilen zudem die Verletzung der kirchlichen Autorität.

Die Funktion der Wundererzählungen im Reliquientraktat ist eine andere. Sie dienen einerseits als argumentative Exempla. Als solche stehen sie in engem Zusammenhang mit Guiberts Forderungen nach einem geregelten Reliquienkult und nach Wahrung der Grabesruhe von Heiligen. Wundererzählungen dienen, ebenfalls als Bestandteile von Argumentationen, auch als ironische Elemente. So verdeutlicht beispielsweise Guiberts Darstellung von Erzählungen aus dem Wunderbuch des Klosters Saint-Médard, daß diese Mittel sind, Distanz zu den Mönchen und ihrer Reliquie zu markieren.

Im Marienlob, das am ehesten den Charakter eines hagiographischen Werkes aufweist, haben die Wundererzählungen Beweisfunktion, indem sie von der Größe Marias zeugen. Sie verfolgen zudem eine didaktische Absicht, indem sie Reue und Buße als Voraussetzung für ein Wunder betonen. Auf die Gnade echter Reue folgt das äußere Gnadenzeichen des Wunders. Die Wundererzählungen im Marienlob können somit in Zusammenhang mit der Seelsorge gestellt werden.

In der Kreuzzugschronik, als historiographischem Werk, erfüllen Erzählungen über Wunder mehrere Funktionen. So beschäftigt sich Guibert wiederholt mit Himmelsphänomenen, die oft Bestandteile historiographischer Texte sind. Diese wunderbaren Zeichen sind offensichtlich an Deutungen gebunden, die ihnen Sinn verleihen. Guiberts Umgang mit Wundererzählungen in den Teilen, die er der Chronik hinzufügt, zeigt, daß er diese Textelemente auf ironische oder ernsthafte Weise verwendet, um Protagonisten seiner Chronik zu charakterisieren. Wunder können also sowohl Elemente sein, über die Kritik geübt werden kann, als auch solche, die Beweiskraft haben. Einzelne Erzählungen über Wunder werden als fabulae, andere als veraces historiae verwendet. In den Teilen der Chronik, die Guibert umarbeitet, verknüpft er Wundererzählungen, über seine Vorlage hinausgehend, mit Themen wie Buße oder Reue.

Wundererzählungen haben in den Werken Guiberts verschiedene Funktionen, die in engem Zusammenhang stehen mit dem Charakter der Werke. Einzelne Erzählungen werden in Guiberts Schriften mehrmals verwendet. Sie können mit unterschiedlichen Botschaften verknüpft werden. Sie dienen als 
demonstrative, als didaktische, als argumentative oder ironische Textelemente und müssen immer in Zusammenhang mit den anderen Elementen der Texte betrachtet werden.

\section{Bezüge}

Im fünften Kapitel werden intertextuelle Bezüge zwischen Wundererzählungen in Guiberts Texten und in weiteren hagiographischen Texten analysiert. Vom Wunderbuch aus Saint-Médard, das Zielscheibe von Guiberts Kritik ist, sind mehrere Versionen bekannt. Ein Vergleich aller bekannten Handschriften des Wunderbuches hat gezeigt, daß es im Laufe des 12. Jahrhunderts mehrmals überarbeitet wurde. Dies in solch großem Ausmaß, daß vielmehr von mehreren Wunderbüchern aus Saint-Médard gesprochen werden muß. Wundererzählungen wurden in den verschiedenen Büchern neu gruppiert, weggelassen oder neu hinzugefügt. Die Veränderungen zeigen, daß die Wunderbücher in einer aktuellen Diskussion standen. Dabei kommt die Konkurrenz geistlicher Institutionen in Soissons zum Ausdruck. Der Vergleich der Schriften zeigt aber auch, daß die Wunderbücher inmitten einer Debatte über Reliquien und deren Wunder standen, an der auch Guibert beteiligt war. So fehlen die von Guibert angegriffenen Wundererzählungen in einer Version von apologetischer Grundhaltung. Die Handschrift dieses Wunderbuchs aus Saint-Médard ist in der reformorientierten Abtei Anchin überliefert, von wo aus SaintMédard reformiert wurde. Daß Guiberts Beitrag zur Debatte um die Reform des Reliquienkultes in Saint-Médard wahrgenommen wurde, ist wiederum über Guiberts Beziehungen zum Kreis reformorientierter Geistlicher zu sehen: Guibert widmet eine seiner späten Schriften dem Abt von Saint-Médard, Geoffroy Cou de Cerf, der die Reform der Abtei ab 1119 vorantrieb. Dieser Abt stand auch mit der Abtei Anchin in Verbindung.

Eine rezeptive Beziehung ist zwischen Marienmirakeln in Guiberts Marienlob und in Marienmirakelsammlungen, die nicht an einen Kultort gebunden sind, festzustellen. Die Analyse der Erzählung des Petrus von Grenoble hat die intertextuellen Beziehungen zwischen den verschiedenen Texten, in denen die Wundererzählung eingefügt ist, deutlich gemacht. Die Erzählung ist in den genannten Marienmirakelsammlungen vielfach überliefert, wobei eine Version aus Guiberts Marienlob einer zweiten in Form eines Briefes gegenübergestellt werden kann. Diese Versionen wurden immer getreulich kopiert. Zwischen den beiden Versionen können enge Beziehungen festgestellt werden. Die Brief-Version der Erzählung bestätigt überdies die Resultate der ersten beiden Kapitel dieser Studie: auch hier wird als Ort der Kommunikation über das Wunder die Synode als Versammlung kirchlicher Würdenträger genannt. Die genannten Geistlichen als Träger der Kommunikation können in Bezug zu denjenigen gesetzt werden, die Guibert erwähnt. In den ortsungebundenen Marienmirakelsammlungen bleiben die Versionen der Erzählungen stabil. Die große Stabilität der Glaubwürdigkeitsgarantien der beiden Versionen erlaubt, die Entstehung und die Kommunikation der Erzählung in Guiberts Umfeld zu 
situieren. Erst in Legendensammlungen des 13. Jahrhunderts wird die narrative Ausgestaltung der Erzählung, wie auch Ort-, Zeit- und Akteurangaben mit größerer Freiheit verändert. Die Erzählung wird nicht mehr als Marienwunder, sondern als Wunder des Heiligen verwendet, der ebenfalls in das Geschehen involviert ist. Der Bezug zu Guiberts Umfeld kann nicht mehr nachvollzogen werden.

Die Untersuchung von Wundererzählungen in den Schriften Guiberts, die nicht im engeren Sinne als hagiographische Texte zu bezeichnen sind, hat gezeigt, daß diese nicht nur dem Lob von Heiligen oder der Propaganda des Kultes einer monastischen oder klerikalen Gemeinschaft dienen. Wundererzählungen sind Elemente verschiedenster Textsorten, innerhalb welcher sie diverse Funktionen wahrnehmen können. Sie nur als Bestandteile einer Gattung "Wunderbuch « zu betrachten, wird dem mittelalterlichen Umgang mit Darstellungen wunderbarer Ereignisse nicht gerecht.

Die Untersuchung hat auch gezeigt, daß Guibert in seinem Umgang mit dem Reliquienkult und den damit in Beziehung stehenden Wundern nicht isoliert betrachtet werden kann. Vielmehr ist immer wieder ein Kreis von reformorientierten Geistlichen sichtbar geworden, innerhalb dessen Guibert über Wunder und Wundererzählungen kommuniziert. Die Analyse von Zeugennennungen für Wunderberichte weist immer wieder auf einen reformorientierten Kreis von monastischen und weltklerikalen Autoritätspersonen hin. Auch die ersten Zusammenstellungen von ortsungebundenen Marienmirakelsammlungen geben Hinweise auf einen Kreis von reformorientierten Mönchen als Initiatoren dieser neuen Textsorte. Ähnliche Überlegungen zum Reliquienkult wie in Guiberts Reliquientraktat sind im Umkreis von Lanfranc und Anselm von Bec/Canterbury belegt. Personen aus diesem Umkreis müssen in Fragen des Kultes Entscheidungen treffen und Reflexionen dazu anstellen. In Guiberts Werken wird deutlich, $\mathrm{da} B$ im Umfeld dieses reformorientierten Personenkreises eine neue Art von Umgang mit Wundererzählungen sichtbar wird: Wundern der heiligen Maria, aber auch anderen Wundern, die wenig oder gar nicht an einen Kultort gebunden sind, werden vor allem didaktische Funktionen zugedacht. Sie haben die Funktion von argumentativen, wie auch von paränetischen Exempla.

Als Ganzes zeigt die Arbeit das Bild eines Abtes aus dem 12. Jahrhundert, der sehr bewußt mit Berichten über wunderbare Ereignisse umgeht. Guibert stellt Überlegungen an zur Deutung von Ereignissen als Wunder und weist in vielen Fällen die Garantien der Glaubwürdigkeit des Berichteten nach. Er schließt Interventionen übernatürlicher Mächte keineswegs aus, versucht aber, sie rational zu fassen und einzuordnen. Wundererzählungen werden je nach Schrift in unterschiedlicher Funktion eingesetzt und in Guiberts intellektuellem und institutionellem Umfeld diskutiert. Dieser Umgang mit dem Wunder widerspricht dem Bild von Guibert als kritisch-rationalem Intellektuellen nicht in dem Maße, wie dies immer wieder postuliert wurde. 
